\title{
Wetting and evaporation of salt-water nanodroplets: A molecular dynamics investigation
}

\author{
Jun Zhang, ${ }^{1, *}$ Matthew K. Borg, ${ }^{2}$ Khellil Sefiane, ${ }^{2,3}$ and Jason M. Reese ${ }^{2}$ \\ ${ }^{1}$ James Weir Fluids Laboratory, Department of Mechanical and Aerospace Engineering, University of Strathclyde, \\ Glasgow G1 1XJ, United Kingdom \\ ${ }^{2}$ School of Engineering, University of Edinburgh, Edinburgh EH9 3FB, United Kingdom \\ ${ }^{3}$ Tianjin Key Laboratory of Refrigeration Technology, Tianjin University of Commerce, Tianjin, China
}

(Received 30 July 2015; published 20 November 2015)

\begin{abstract}
We employ molecular dynamics simulations to study the wetting and evaporation of salt-water nanodroplets on platinum surfaces. Our results show that the contact angle of the droplets increases with the salt concentration. To verify this, a second simulation system of a thin salt-water film on a platinum surface is used to calculate the various surface tensions. We find that both the solid-liquid and liquid-vapor surface tensions increase with salt concentration and as a result these cause an increase in the contact angle. However, the evaporation rate of salt-water droplets decreases as the salt concentration increases, due to the hydration of salt ions. When the water molecules have all evaporated from the droplet, two forms of salt crystals are deposited, clump and ringlike, depending on the solid-liquid interaction strength and the evaporation rate. To form salt crystals in a ring, it is crucial that there is a pinned stage in the evaporation process, during which salt ions can move from the center to the rim of the droplets. With a stronger solid-liquid interaction strength, a slower evaporation rate, and a higher salt concentration, a complete salt crystal ring can be deposited on the surface.
\end{abstract}

DOI: 10.1103/PhysRevE.92.052403

PACS number(s): 68.08.-p, 47.11.Mn

\section{INTRODUCTION}

Salt water is one of the most abundant resources on this planet and we need to find ways of using it to help solve current social, health, environmental, and economic problems. One promising opportunity is to produce fresh water from salt water using desalination technologies, such as distillation, membrane processes, and solar desalination $[1,2]$. Another is cooling electricity-generating equipment in the thermoelectric power industry. However, salt water is also well known as a cause of structural and environmental problems, such as corrosion and salt weathering [3]. The latter is an erosion process that deteriorates natural stone and building materials and results from the crystallization of salt. Salt may naturally exist inside stone or be present in precipitations, such as acid rain. Used as building materials, most stones are subject to wetting and evaporation cycles; during the latter, salts initially dissolved in water may crystallize. Repeated wetting and evaporation and the resultant stresses can eventually lead to physical breakdown of the stone. In both applications and problems, a fundamental fluid question is therefore the wetting and evaporation properties of salt-water droplets.

Research interest in salt-water droplets has triggered experimental investigations. Sghaier et al. [4] reported that the contact angle of salt-water droplets on hydrophilic surfaces significantly increases with the salt concentration. ShahidzadehBonn et al. [5,6] studied the forms of salt crystal deposited after evaporation. However, current experimental techniques cannot provide high-resolution dynamical information down to

\footnotetext{
" zhangjun04@foxmail.com
}

Published by the American Physical Society under the terms of the Creative Commons Attribution 3.0 License. Further distribution of this work must maintain attribution to the author(s) and the published article's title, journal citation, and DOI. the molecular scale. To find ways to reduce damage caused by salt weathering and to enhance the efficiency of desalination, a better understanding of the molecular mechanism underlying the wetting and evaporation of salt-water droplets on surfaces is highly desirable.

Molecular dynamics (MD) is a computational tool that can provide useful nanoscale material and fluid predictions. It has been successfully applied to study the wetting and evaporation of a variety of nanodroplets. For example, Werder et al. [7] investigated the static wetting properties of pure-water droplets on graphitic surfaces. Ritos et al. [8] investigated the dynamic wetting of water droplets on moving surfaces. Zhang et al. $[9,10]$ studied the evaporation of argon droplets and reported the dependence of the evaporation mechanism on the curvature of the contact line. Chen et al. [11] investigated the evaporation of Lennard-Jones fluid droplets containing rigid particles and predicted the aggregate structure in the evaporative growth processes. These results showed similarities between the behavior of macrodroplets and nanodroplets.

However, so far, studies of salt-water droplets using MD have been scarce. For wetting, Daub et al. [12] reported that the contact angles of salt-water nanodroplets increase with the salt concentration. For evaporation, only salt-water clusters in periodic boxes have been investigated, without contact with solid surfaces [13,14]. These studies showed that the evaporation rates of clusters containing salt ions are slower than pure-water clusters.

In this paper we employ MD simulations to study the wetting and evaporation of salt-water droplets, specifically, aqueous sodium chloride $(\mathrm{NaCl})$ nanodroplets on smooth platinum surfaces. Our aim is to investigate the effect of salt concentration on the equilibrium contact angle and the evaporation rate and understand the inherent physical phenomena. Importantly, we are interested in the forms of the salt crystals deposited after evaporation and the crucial factors determining whether a clump or a ringlike crystal is deposited. 


\section{SIMULATION MODEL AND INTERATOMIC POTENTIALS}

We perform MD simulations of salt-water nanodroplets on platinum surfaces. We use the mdFOAM software [8,15-19], which is a highly parallelized and GPU-accelerated nonequilibrium MD solver implemented within the open source framework OpenFOAM [20]. In this section the simulation system, the interatomic potential function and parameters, the technique for measuring flow fields and contact angles, and the method for calculating surface tensions are described.

\section{A. Simulation system}

Figure 1 shows how we prepare the simulation of a saltwater droplet on a smooth platinum surface. An equilibrium MD simulation of a fully periodic cubic box (of dimension 5.68 $\mathrm{nm}$ ) containing 5832 pure-water molecules is performed first, as shown in the top left in Fig. 1. Using the FADE mass-stat technique of Borg et al. [21], we insert $\mathrm{Na}^{+}$and $\mathrm{Cl}^{-}$ions and delete water molecules to reach a target salt concentration. The system is equilibrated and then the salt-water box is placed on an atomic platinum surface. The full simulation box is then $18.8 \mathrm{~nm}$ in the $x$ and $z$ directions (parallel to the solid surface) and $31.4 \mathrm{~nm}$ in the $y$ direction (normal to the solid surface). Eight layers of platinum atoms in an fcc lattice (lattice constant $3.92 \AA$ ) are used to simulate the solid surface. The atoms in the bottom four layers are fixed, while the atoms in the top four layers are coupled to a Berendsen thermostat to control the temperature of the substrate. Periodic boundary conditions are applied in all three directions. The integration time step is $2 \mathrm{fs}$. This full MD system is run for $2 \mathrm{~ns}$ of problem time at $300 \mathrm{~K}$ to reach an equilibrium state and the formation of a nanodroplet on the surface. After steady state the simulation is run for another $2 \mathrm{~ns}$ of averaging time in order to measure the density contours and the equilibrium contact angle.

Finally, in order to study the evaporation properties, the temperature of the platinum surface is increased in time to $600 \mathrm{~K}$ using a ramp function. The liquid water molecules in the droplets continuously evaporate to form a vapor, while all $\mathrm{Na}^{+}$and $\mathrm{Cl}^{-}$ions remain part of the droplet, thus increasing the salt concentration and ultimately reaching a saturation point that leaves salt crystal deposits on the surface.

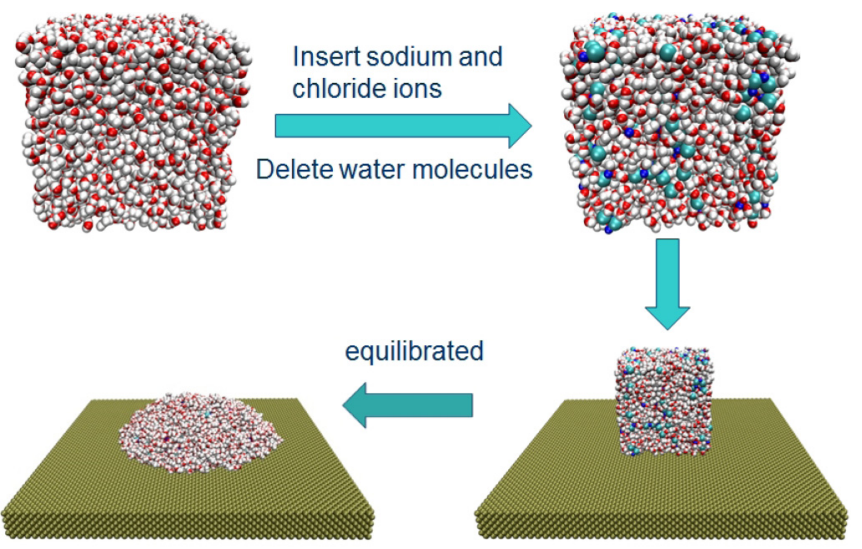

FIG. 1. (Color online) Routine for preparing the MD simulation system of a salt-water nanodroplet on a platinum surface.

\section{B. Intermolecular potentials}

Intermolecular potentials are needed for all four species in these MD simulations: water molecules $\left(\mathrm{H}_{2} \mathrm{O}\right)$, sodium ions $\left(\mathrm{Na}^{+}\right)$, chloride ions $\left(\mathrm{Cl}^{-}\right)$, and platinum atoms $(\mathrm{Pt})$. The rigid TIP4P/2005 model [22] is used to simulate water molecules. This model consists of four sites: one oxygen site (no charge), two hydrogen sites $(0.5564 e)$, and one massless $\mathrm{M}$ site $(-1.1128 e)$ located at $0.1546 \AA$ from the oxygen atom along the bisector of the hydrogen atoms. Hamilton's quaternions are used in order to keep the fixed geometry of the water molecules, with an $\mathrm{O}-\mathrm{H}$ bond distance of $0.9572 \AA$ and an $\mathrm{H}-\mathrm{O}-\mathrm{H}$ angle of $104.52^{\circ}$. The $\mathrm{Na}^{+}(1.0 e)$ and $\mathrm{Cl}^{-}(-1.0 e)$ ions are treated as nonpolarizable sites with fixed charges. All intermolecular interactions between pairs of molecules for $\mathrm{O}, \mathrm{H}, \mathrm{M}, \mathrm{Na}^{+}, \mathrm{Cl}^{-}$, and $\mathrm{Pt}$ sites are based on the following combined Lennard-Jones (LJ) and Coulomb potential:

$$
u_{\alpha \beta}(r)=4 \varepsilon_{\alpha \beta}\left[\left(\frac{\sigma_{\alpha \beta}}{r}\right)^{12}-\left(\frac{\sigma_{\alpha \beta}}{r}\right)^{6}\right]+\frac{1}{4 \pi \varepsilon_{0}} \frac{q_{\alpha} q_{\beta}}{r},
$$

where $r$ is the distance between site $\alpha$ and site $\beta, q_{\alpha}$ is the electric charge associated with site $\alpha, \varepsilon_{0}$ is the permittivity of vacuum, $\varepsilon_{\alpha \beta}$ is the well depth of the LJ potential, and $\sigma_{\alpha \beta}$ is the characteristic diameter. The LJ parameters and charges for each site are listed in Table I. The interaction parameters for the $\mathrm{H}, \mathrm{O}$, and $\mathrm{M}$ sites of the water model are from Ref. [22], while those for $\mathrm{Na}^{+}$and $\mathrm{Cl}^{-}$ions are from Ref. [23]. Coulomb interactions are between sites with charges and the Coulombic force potential is truncated and shifted at the same cutoff distance of $1.2 \mathrm{~nm}$ as in the LJ interactions. Compared with other methods, this approach can provide a balance of acceptable accuracy with computational efficiency $[7,8]$.

For the LJ cross interactions between two different sites, the Lorentz-Berthelot mixing rules are used to determine $\sigma_{\alpha \beta}$ and $\varepsilon_{\alpha \beta}$, i.e.,

$$
\begin{gathered}
\sigma_{\alpha \beta}=\frac{\sigma_{\alpha}+\sigma_{\beta}}{2}, \\
\varepsilon_{\alpha \beta}=\lambda \sqrt{\varepsilon_{\alpha} \times \varepsilon_{\beta}},
\end{gathered}
$$

where $\lambda$ is a parameter to tune the interaction strength. For the interactions between the sites in aqueous $\mathrm{NaCl}$ droplets, $\lambda=$ 1.0. According to this rule, there are no $\mathrm{LJ}$ cross interactions between an $\mathrm{H}$ site and the $\mathrm{Na}^{+}$and $\mathrm{Cl}^{-}$ion sites, because the interaction strength for $\mathrm{H}$ is zero. However, as reported in Ref. [23], it is important to add an extra LJ potential

TABLE I. Interatomic potential parameters for sites in water molecules, $\mathrm{Na}^{+}$and $\mathrm{Cl}^{-}$ions, and $\mathrm{Pt}$ atoms.

\begin{tabular}{lclc}
\hline \hline Site & $\varepsilon(\mathrm{kJ} / \mathrm{mol})$ & $\sigma(\AA)$ & $q(e)$ \\
\hline $\mathrm{H}$ & 0 & 0 & 0.5564 \\
$\mathrm{O}$ & 0.775 & 3.1589 & 0 \\
$\mathrm{M}$ & 0 & 0 & -1.1128 \\
$\mathrm{Na}^{+}$ & 0.196 & 2.45 & 1.0 \\
$\mathrm{Cl}^{-}$ & 0.628 & 4.1 & -1.0 \\
$\mathrm{H}_{-} \mathrm{Na}^{+}$ & 0.180 & 1.55 & \\
$\mathrm{H}-\mathrm{Cl}^{-}$ & 0.323 & 2.375 & 0 \\
$\mathrm{Pt}$ & 66.84 & 2.471 & \\
\hline \hline
\end{tabular}


between $\mathrm{H}$ sites in water and $\mathrm{Cl}^{-}$sites in order to prevent them from getting too close (which causes an overestimation of ion hydration). The modified $\mathrm{LJ}$ potential parameters for $\mathrm{H}-\mathrm{Cl}^{-}$and $\mathrm{H}-\mathrm{Na}^{+}$pairs are listed in Table I.

The interactions between platinum atoms and all other sites in the droplets are based on the Lorentz-Berthelot mixing rules for $\mathrm{Pt}-\mathrm{O}, \mathrm{Pt}_{-\mathrm{Na}}{ }^{+}$, and $\mathrm{Pt}-\mathrm{Cl}^{-}$pairs; the $\mathrm{LJ}$ parameters for $\mathrm{Pt}-\mathrm{Pt}$ [24] are also listed in Table I. Considering the hydrophilic properties of water droplets on platinum surfaces, we choose two values for the parameter $\lambda, 0.25$ or 0.38 , to represent a partial wetting or a complete wetting surface, respectively, for pure-water droplets.

\section{Measuring flow fields and contact angles}

Flow property fields of the droplets, such as density and velocity, are obtained on-the-fly from the MD simulation. To do this, we use a cylindrical coordinate system $(r, \phi, y)$ that has the topmost solid surface layer as its zero reference level for the $y$ direction and a normal line through the center of mass of the droplet as its reference axis. Considering the axial symmetry of droplets, the three-dimensional data are projected into two-dimensional bins $(r, y)$ by averaging in concentric rings. To extract the microscopic contact angle from the density contours, a popular method is to fit the liquid-vapor dividing interface to a circle $[7,8]$. Here the liquid-vapor interface is defined by the points in the two-dimensional bins that have half the bulk density of the liquid phase. A circular fit is then made through these points and extrapolated to the solid-liquid dividing interface, which is defined as the positions of the topmost solid atoms. Note that the points below a height of $7.5 \AA$ from the solid surface are not taken into account for the fit, in order to avoid the influence of density ordering within the solid-liquid interface region. The contact angle is then measured using the tangent to the fitting circle at the solid-liquid dividing interface, as shown in Fig. 2.

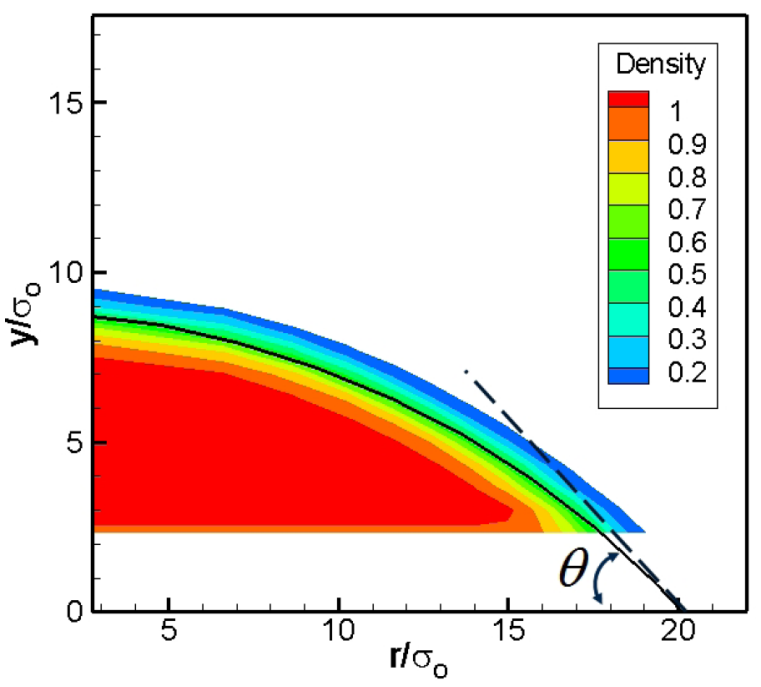

FIG. 2. (Color online) Density contours for droplets and contact angle measurements. The black solid line is obtained by applying a circular fit to the points with half of the bulk liquid density. The coordinates and density are normalized by $\sigma_{\mathrm{O}}$ (the characteristic diameter of the oxygen site in water molecules) and $\sigma_{\mathrm{O}}^{-3}$, respectively.

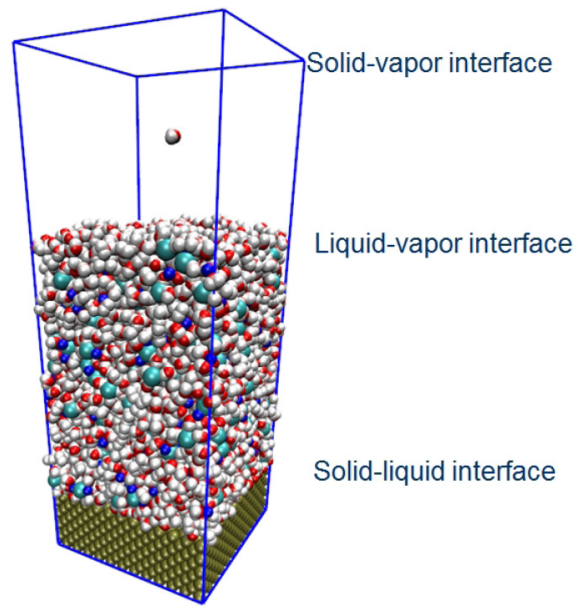

FIG. 3. (Color online) The MD setup for the calculation of surface tensions. The platinum atoms are fixed at the bottom of the simulation box and the thin film of salt water covers the solid surface. After the system is equilibrated, there are three interfaces formed from bottom to top: solid-liquid, liquid-vapor, and solid-vapor (due to the periodic boundary conditions).

\section{Surface tension calculations}

A second MD simulation system, shown in Fig. 3, is also set up in order to calculate the individual solid-liquid, liquid-vapor, and solid-vapor surface tensions. The simulation box is $4.7 \mathrm{~nm}$ in the $x$ and $z$ directions (parallel to the solid surface) and $12.6 \mathrm{~nm}$ in the $y$ direction (normal to the solid surface). A thin salt-water film of $7.5 \mathrm{~nm}$ depth covers the platinum surface. The platinum atoms are fixed at the lattice sites, while the water molecules and $\mathrm{Na}^{+}$and $\mathrm{Cl}^{-}$ions are free to move at a fluid temperature of $300 \mathrm{~K}$ using the Berendsen thermostat. Periodic boundary conditions are applied in all three directions. The interatomic potential parameters are the same as for the droplet simulations. After the system is equilibrated, three interfaces (solid-liquid, liquid-vapor, and solid-vapor) are formed. The surface tensions $\gamma$ are obtained by integrating local stress tensor components along the $y$ direction (i.e., normal to the interfaces):

$$
\gamma=\int\left[P_{n}(y)-P_{t}(y)\right] d y,
$$

where $P_{n}(y)$ and $P_{t}(y)$ are the stresses normal and tangential to an interface, respectively. Note that in our simulation system the normal stress is the same as the saturated vapor pressure, due to mechanical equilibrium in the $y$ direction. Because the vapor pressure of salt water is very low at $300 \mathrm{~K}$, the contribution of normal stress to the surface tension is negligible when compared to the contribution of tangential stress. To obtain the local tangential stress, the simulation box is divided into bins in the $y$ direction. Considering that the solid surface extends infinitely, the tangential contribution from the fluidsolid interaction vanishes [25]. In each bin, the tangential stress is calculated as

$$
P_{t}(y)=k_{B} T \rho(y)-\frac{1}{4 A}\left\langle\sum_{i \neq j} \frac{x_{i j}^{2}+z_{i j}^{2}}{r_{i j}} \frac{\partial U_{f f}\left(r_{i j}\right)}{\partial r_{i j}} \delta\left(y-y_{i}\right)\right\rangle,
$$


where $k_{B}$ is the Boltzmann constant; $T$ is the temperature; $\rho(y)$ is the number density; $A$ is the surface area of the bin; $x_{i}, y_{i}$, and $z_{i}$ are the components of the position of the water molecules, $\mathrm{Na}^{+}$, or $\mathrm{Cl}^{-}$ions at $\mathbf{r}_{i} ; r_{i j}=\left|\mathbf{r}_{i}-\mathbf{r}_{j}\right| ; U_{f f}\left(r_{i j}\right)$ is the interatomic potential between two molecules; and angular brackets denote an ensemble average. For the liquid-vapor surface tension, the integration in Eq. (4) is from the liquid bulk region to the vapor bulk region, while for the solid-liquid and solid-vapor surface tensions the integration region is limited to the region containing fluid molecules.

\section{RESULTS AND DISCUSSION}

\section{A. Wetting}

To study comprehensively the wetting properties of saltwater droplets on platinum surfaces, two solid-liquid interaction strength parameters $(\lambda=0.25$ and 0.38$)$ are used, as explained in Sec. II B. Five different concentrations of the salt-water droplets are studied, as well as pure-water droplets. The numbers of water molecules and $\mathrm{Na}^{+}$and $\mathrm{Cl}^{-}$ions in the different nanodroplets are shown in Table II. The salt mass concentration is defined as

$$
c=\frac{m_{\mathrm{Na}^{+}}+m_{\mathrm{Cl}^{-}}}{m_{\mathrm{water}}+m_{\mathrm{Na}^{+}}+m_{\mathrm{Cl}^{-}}} \times 100 \%,
$$

where $m_{\mathrm{Na}^{+}}, m_{\mathrm{Cl}^{-}}$, and $m_{\text {water }}$ are the masses of the $\mathrm{Na}^{+}$ions, $\mathrm{Cl}^{-}$ions, and water molecules, respectively, in the droplets.

For each droplet case, the MD simulations are first run for $2 \mathrm{~ns}$ of problem time to ensure that the system relaxes to an equilibrium state at $300 \mathrm{~K}$. Sampling in cylindrical bins provides the liquid density contours and the contact angles are then determined as outlined above. Table III lists the resulting contact angles of salt-water droplets for different salt concentrations and solid-liquid interaction strength parameters. It can be seen that the contact angle increases with the salt concentration; this phenomenon has been reported by experiments [4] and other MD results [12] in the literature. Note that in the results for $\lambda=0.38$, pure-water droplets and droplets with the lowest salt concentration almost completely wet the surface, so it is difficult to measure the contact angle quantitatively using a circle fit to the liquid-vapor interface.

Besides analyzing the density contours of droplets obtained from the MD simulations, the contact angle can also be determined from Young's equation

$$
\cos \left(\theta_{Y}\right)=\frac{\gamma_{s v}-\gamma_{s l}}{\gamma_{l v}}
$$

TABLE II. Number of water molecules and $\mathrm{Na}^{+}$and $\mathrm{Cl}^{-}$ions in nanodroplets for each simulation case. The corresponding salt mass concentrations are given in the last column.

\begin{tabular}{lcrrc}
\hline \hline Case & $N_{\text {water }}$ & $N_{\mathrm{Na}^{+}}$ & $N_{\mathrm{Cl}^{-}}$ & Salt concentration \\
\hline 1 & 5832 & 0 & 0 & 0 \\
2 & 5632 & 100 & 100 & $5.4 \%$ \\
3 & 5432 & 200 & 200 & $10.7 \%$ \\
4 & 5232 & 300 & 300 & $15.7 \%$ \\
5 & 5032 & 400 & 400 & $20.5 \%$ \\
6 & 4832 & 500 & 500 & $25.1 \%$ \\
\hline \hline
\end{tabular}

TABLE III. Contact angles of salt-water nanodroplets for different salt concentrations and solid-liquid interaction strength parameters.

\begin{tabular}{lcc}
\hline \hline $\begin{array}{l}\text { Salt } \\
\text { concentration }\end{array}$ & $\begin{array}{c}\text { Contact angle } \\
(\lambda=0.25)\end{array}$ & $\begin{array}{r}\text { Contact angle } \\
(\lambda=0.38)\end{array}$ \\
\hline 0 & $43.6^{\circ}$ & \\
$5.4 \%$ & $46.7^{\circ}$ & $15.0^{\circ}$ \\
$10.7 \%$ & $51.8^{\circ}$ & $19.9^{\circ}$ \\
$15.7 \%$ & $55.2^{\circ}$ & $28.7^{\circ}$ \\
$20.5 \%$ & $59.2^{\circ}$ & $36.1^{\circ}$ \\
$25.1 \%$ & $60.8^{\circ}$ & \\
\hline \hline
\end{tabular}

where $\theta_{Y}$ is Young's contact angle and $\gamma_{s v}, \gamma_{s l}$, and $\gamma_{l v}$ are the solid-vapor, solid-liquid, and liquid-vapor surface tensions, respectively. In order to understand the phenomenon of the contact angle increasing with salt concentration, we investigated thin salt-water films on platinum surfaces (for details refer to Sec. II D and Fig. 3). After the system is equilibrated, density and pressure distributions are sampled in bins along the $y$ direction. Figure 4 shows the density distribution of water molecules and $\mathrm{Na}^{+}$and $\mathrm{Cl}^{-}$ions along the $y$ direction for the case with a salt concentration of $10.7 \%$ and $\lambda=0.25$. Note that the quantities for the $\mathrm{Na}^{+}$and $\mathrm{Cl}^{-}$ ions are enlarged by 10 times in Fig. 4 for clear comparison. It is evident that $\mathrm{Na}^{+}$and $\mathrm{Cl}^{-}$ions tend to migrate away from the solid-liquid and liquid-vapor interfaces. This characteristic of salt ions is the opposite of the preferential nature of surfactants, such as alcohol, to stick to interfaces.

By integrating the difference between the normal and tangential stresses [as in Eq. (4)] through each interface region the three surface tensions are obtained. Note that the liquid-vapor surface tension obtained here is an absolute value, while the solid-liquid and solid-vapor surface tensions are values relative to a solid-vacuum interface [26]. In our

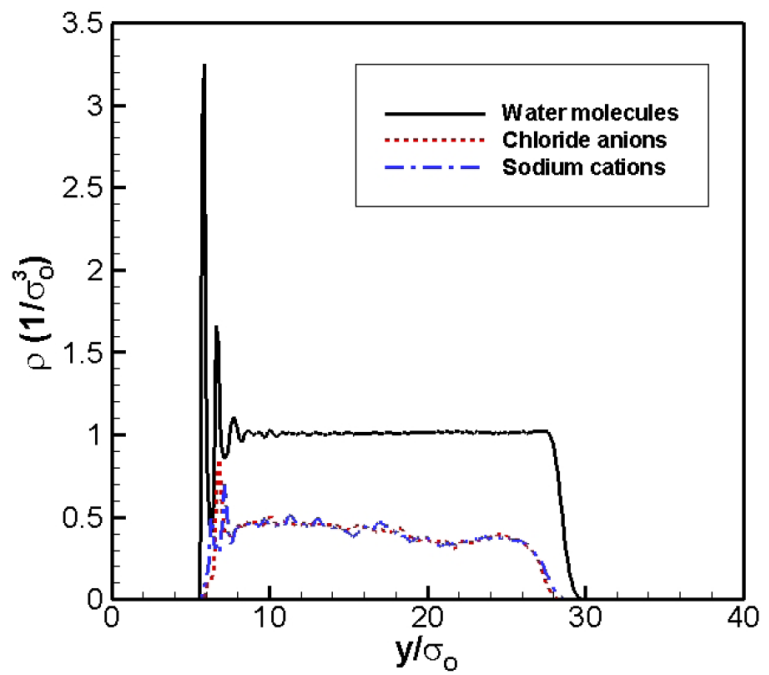

FIG. 4. (Color online) Distribution of water molecules and $\mathrm{Na}^{+}$ and $\mathrm{Cl}^{-}$ions along the $y$ direction in the thin-film case. The overall salt concentration is $10.7 \%$ and the solid-liquid interaction strength parameter is 0.25 . The densities of $\mathrm{Na}^{+}$and $\mathrm{Cl}^{-}$ions are increased by a factor of 10 for a clear comparison. 
TABLE IV. Surface tensions of solid-liquid and liquid-vapor interfaces for different salt concentrations and solid-liquid interaction strength parameters.

\begin{tabular}{lccc}
\hline \hline $\begin{array}{l}\text { Salt } \\
\text { concentration }\end{array}$ & $\begin{array}{c}\text { Solid-liquid surface tension } \\
(\lambda=0.25)(\mathrm{mN} / \mathrm{m})\end{array}$ & $\begin{array}{c}\text { Solid-liquid surface tension } \\
(\lambda=0.38)(\mathrm{mN} / \mathrm{m})\end{array}$ & $\begin{array}{c}\text { Liquid-vapor surface } \\
\text { tension }(\mathrm{mN} / \mathrm{m})\end{array}$ \\
\hline 0 & -40.4 & & 63.9 \\
$5.4 \%$ & -38.4 & & 64.5 \\
$10.7 \%$ & -37.2 & -66.1 & 66.8 \\
$15.7 \%$ & -35.1 & -65.1 & 67.8 \\
$20.5 \%$ & -31.5 & -64.6 & 69.4 \\
$25.1 \%$ & -30.1 & -63.4 & 72.0 \\
\hline \hline
\end{tabular}

simulation cases, the saturated vapor pressure is very low, so the solid-vapor surface tensions are almost zero. The results for the solid-liquid and liquid-vapor surface tensions are listed in Table IV for different salt concentrations and solid-liquid interaction strength parameters.

Specifically, our MD simulations predict that the surface tension of pure water is around $63.9 \mathrm{mN} / \mathrm{m}$ at $300 \mathrm{~K}$, which is slightly smaller than the experimental result $(71.7 \mathrm{mN} / \mathrm{m})$ and the $\mathrm{MD}$ result $(68.4 \mathrm{mN} / \mathrm{m})$ obtained by Alejandre and Chapela [27] using the same water model but with Ewald summation for the long-range force calculations. This deviation may be caused by the truncation of both the LJ and the electrostatic potentials at $1.2 \mathrm{~nm}$ in our MD calculations. However, this deviation does not affect the comparison of contact angles determined from Young's equation and from the density contours of the droplets, as we use the same truncation distance in all our simulations.

From the data in Table IV we can conclude that both the solid-liquid and liquid-vapor surface tensions increase with the salt concentration. To further benchmark our simulation results, we compare the increments of liquid-vapor surface tension as a function of salt concentration with experimental results [28], as shown in Fig. 5. There is reasonable agreement. Note that in our simulation model, the thickness of the liquid film is about $6 \mathrm{~nm}$ and the calculated surface tensions are independent of film thickness [29].

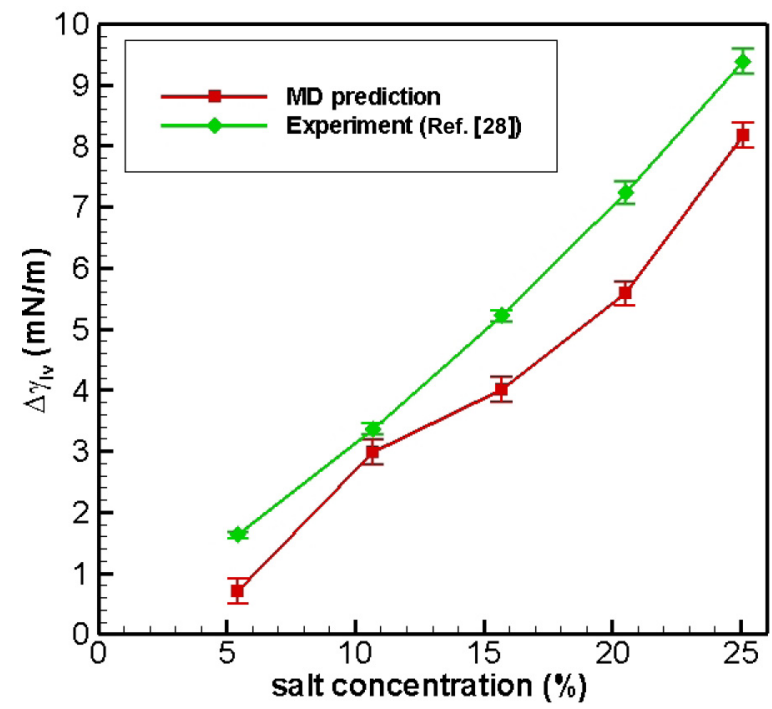

FIG. 5. (Color online) Relation of the increments of the liquidvapor surface tension to the salt concentration.
With the surface tensions determined, Young's contact angle can be calculated using Eq. (7). Figure 6 shows Young's contact angles and the measured MD contact angles from the density contours of nanodroplets for different salt concentrations. The predicted Young's angle confirms that the contact angle of salt-water droplets increases with salt concentration. This is because both the solid-liquid and liquidvapor surface tensions increase with salt concentration. Note that there are some quantitative deviations between Young's contact angle and the measured contact angle. There are two possible reasons for this. The first is the calculation method for the surface tensions: a more rigorous way to calculate solid-liquid and liquid-vapor surface tensions would be to perform two separate simulations [30] and consider the effect of the tangential stress caused by the solid surface [31]. The second is that Young's equation is derived on the macroscopic scale, but the radii of the droplets in our simulations are only several nanometers; on such a small scale, the line tension may play an important role. Similar to the definition of surface tension, line tension is the excess free energy per unit length of a three-phase contact line [32]. There is evidence that the contact angle of nanodroplets on solid surfaces could deviate from that predicted by Young's equation [7,33-35]. A possible modification to Young's equation for nanodroplets

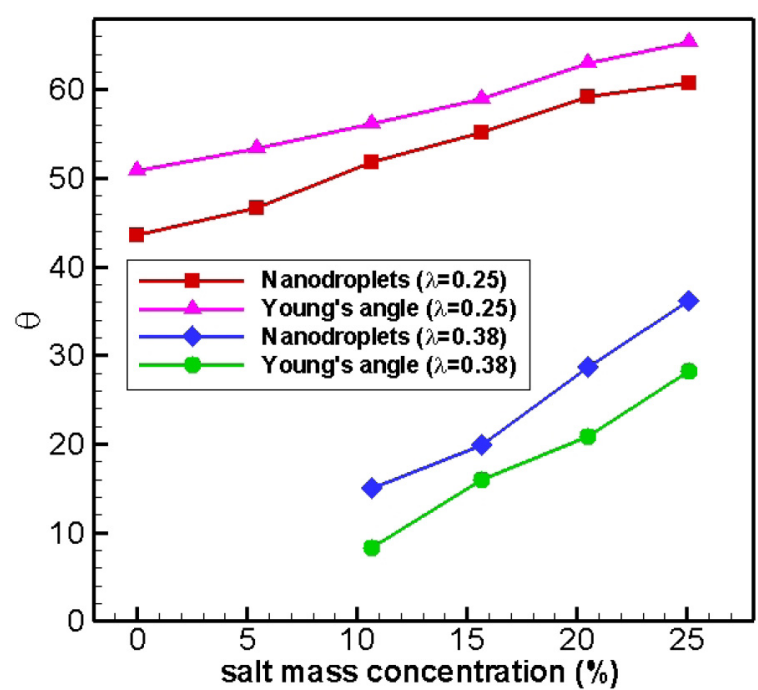

FIG. 6. (Color online) Contact angle determined from the density contours of nanodroplets and from Young's equation. In Young's equation, the surface tensions are obtained from MD measurements in our system of a salt-water film covering a platinum surface. 
incorporates the effect of line tension [36]. An alternative point of view is that the increase of pressure in nanodroplets due to the Kelvin effect [37] results in a difference in adsorption at the solid-liquid interface and hence in the corresponding surface tension. This has been proposed to explain the size dependence of the contact angle [38,39]. However, here we do not quantitatively study the effect of line tension and the change of surface tension but only use Young's equation to uncover why the contact angle of salt-water nanodroplets increases with salt concentration.

\section{B. Evaporation}

Our aim in simulating the evaporation of salt-water nanodroplets is to investigate how the evaporation rate changes with the salt concentration and how the salt crystals deposit on the surface after the water is evaporated. An interesting phenomenon in the evaporation of water droplets containing particles is the so-called coffee-ring effect, where the remaining solid forms into a ringlike shape. Deegan et al. [40-42] proposed that capillary flow, induced by droplet evaporation at a pinned contact line, is the main cause of the coffee-ring phenomenon. Our simulation results (below) agree with this, but we also show that the forms of salt crystals that are deposited on the surface are dependent on the solid-liquid interaction strength, the evaporation rate, and the salt concentration.

\section{Results for solid-liquid interaction strength parameter $\lambda=0.25$}

When the simulation system has reached steady state at $300 \mathrm{~K}$ for $4 \mathrm{~ns}$ of problem time, the temperature of the platinum surface is increased from 300 to $600 \mathrm{~K}$ in $1 \mathrm{~ns}$ of problem time, i.e., the temperature ramp rate is $300 \mathrm{~K} / \mathrm{ns}$. Due to heat transfer between the platinum surface and the droplet, the temperature of the droplet increases and water molecules escape from the condensed liquid phase to become vapor. To analyze the evaporation rate, we use the criterion proposed by Maruyama et al. [43] to define whether a water molecule belongs to the liquid or vapor phase. This criterion is based on the number of neighboring water molecules within a certain distance: two water molecules are considered neighbors if the distance between them is less than $6.3 \AA$. A water molecule is identified as part of the liquid phase if its number of neighbors is larger than 10; otherwise it is identified as being part of the vapor phase.

Figure 7 shows the evolution in time of the number of vapor molecules for three different initial concentrations of salt-water nanodroplets with a ramp rate of $300 \mathrm{~K} / \mathrm{ns}$. For pure water droplets, all of the water molecules undergo a phase change by $5.8 \mathrm{~ns}$, while for salt-water droplets with concentrations of $10.7 \%$ and $20.5 \%$ this occurs at about 6.4 and $6.8 \mathrm{~ns}$, respectively. This means that the evaporation rate of salt-water droplets decreases as the salt concentration increases. Similar trends have been reported for water clusters containing salt ions by Caleman and van der Spoel [13] and Wang et al. [14].

This phenomenon could be understood from the microscopic point of view as follows. Due to electrostatic interactions, the oxygen atoms in water molecules are attracted to positively charged $\mathrm{Na}^{+}$ions, while the hydrogen atoms in

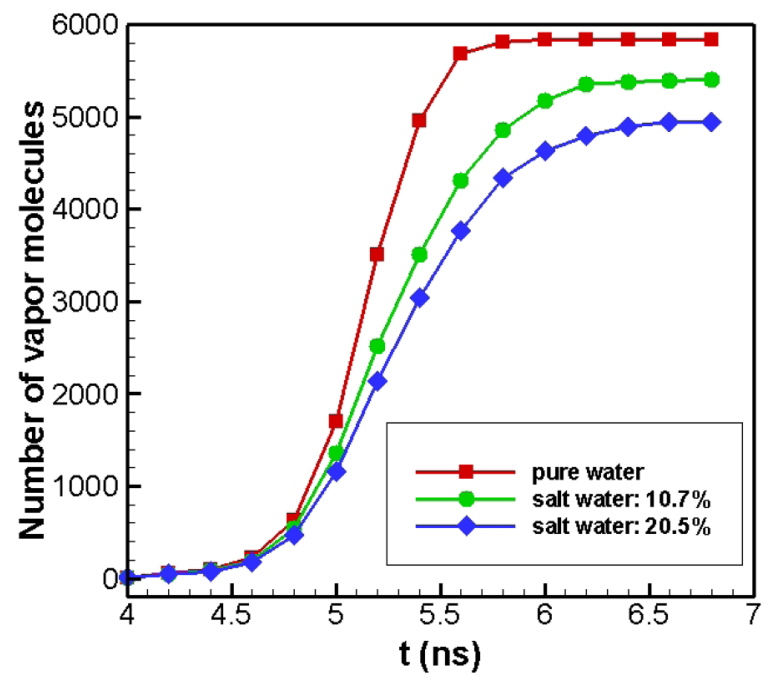

FIG. 7. (Color online) Temporal evolution of the number of vapor molecules for three different concentrations of salt-water droplets. The surface temperature is raised from 300 to $600 \mathrm{~K}$ in the period from 4 to $5 \mathrm{~ns}$.

water molecules are attracted to negatively charged $\mathrm{Cl}^{-}$ions. So $\mathrm{Na}^{+}$and $\mathrm{Cl}^{-}$ions are surrounded by a hydration shell of regularly arranged water molecules. The interaction strength between salt ions and water molecules is stronger than that between water molecules, where there is only weak hydrogen bonding. The $\mathrm{Na}^{+}$and $\mathrm{Cl}^{-}$ions themselves are nonvolatile, so their presence slows the evaporation of water molecules. In terms of thermodynamics, the saturated vapor pressure decreases as the salt concentration increases [44] and a lower vapor pressure results in a slower evaporation rate.

The evaporation modes for pure-water and salt-water droplets are also different. Figures 8(a) and 8(b) show the temporal evolution of the liquid-vapor interface during the evaporation of pure-water and salt-water droplets with a concentration of $20.5 \%$. For the pure-water droplets, there are three stages [Fig. 8(a)]. The first is from 4.0 to $4.6 \mathrm{~ns}$, during which the evaporation rate is slow (Fig. 7) and the droplets spread out a little due to the increase in temperature. The second stage is from 4.6 to $5.0 \mathrm{~ns}$, during which the evaporation becomes quicker and the liquid-vapor interface is pinned at the contact line. As shown in Fig. 8(c), there is during this stage a flow from the center to the rim of the droplet to compensate for the loss of liquid molecules due to evaporation at the contact line. This evaporation mode is usually called the constant contact radius mode $[45,46]$ and has been previously reported in experiments for large droplets [40-42]. After $5.0 \mathrm{~ns}$, the droplet shrinks along the surface until all of the liquid water molecules change to vapor; this is the third stage.

On the other hand, for salt-water droplets there are only two observed stages in the evaporation process [Fig. 8(b)]. The first is the same as that for pure-water droplets, from 4.0 to $4.8 \mathrm{~ns}$. In the second stage, the droplet slips along the surface during evaporation. What is evident from the velocity field measurements is that, unlike our simulations of pure-water droplet evaporation, there is no distinct outward flow within the droplet at $5 \mathrm{~ns}$ [Fig. 8(d)]. Further simulations are required to better resolve this velocity field due to the thermal fluctuations, 
(a)
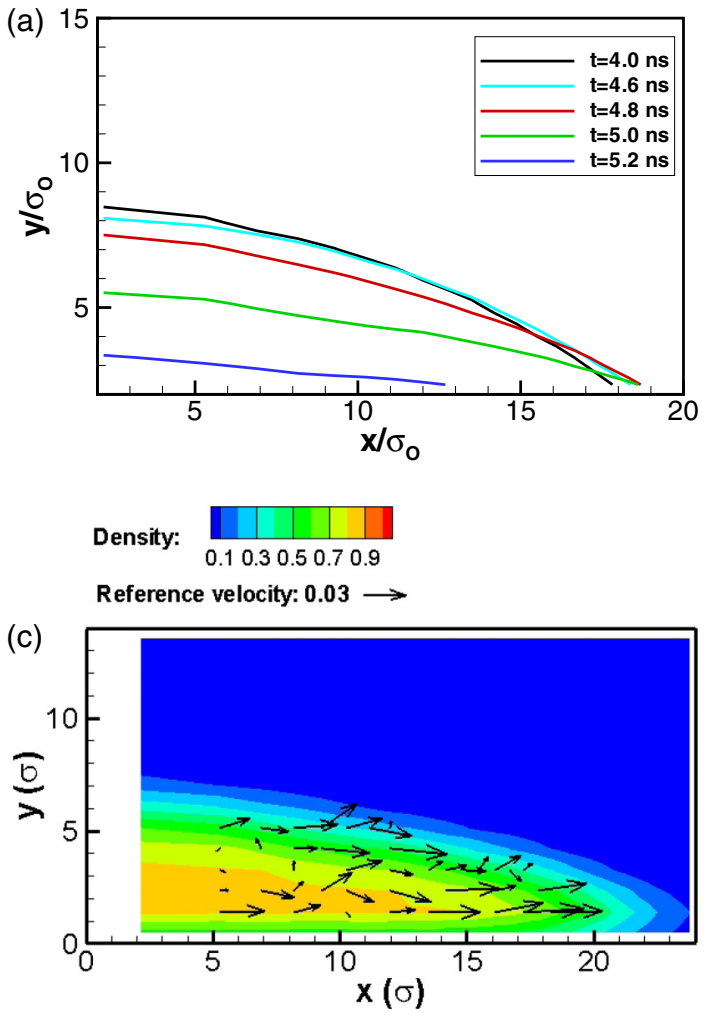

(b)

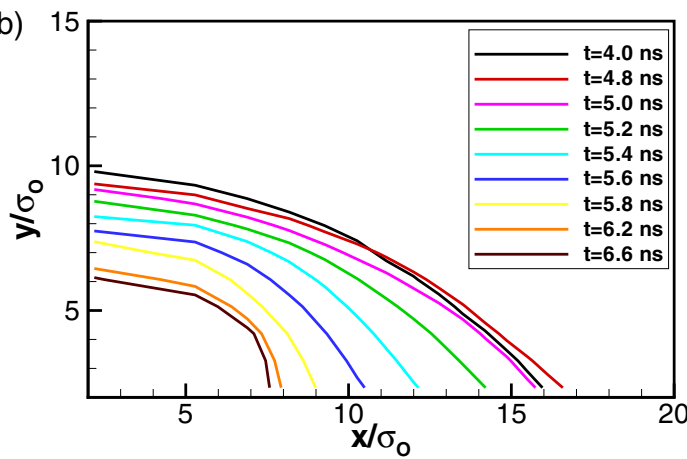

Density: 0.10 .30 .50 .70 .9

Reference velocity: $0.03 \rightarrow$

(d)

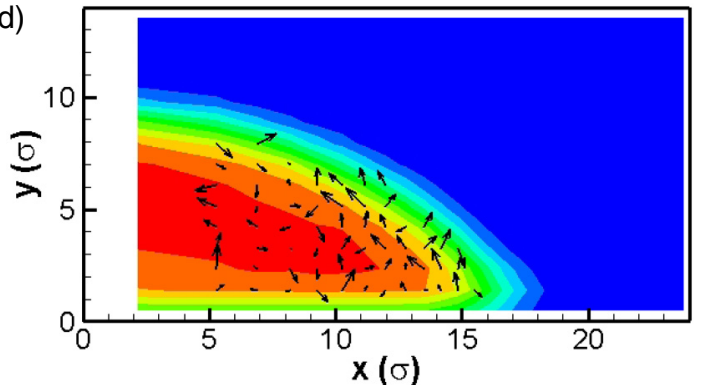

FIG. 8. (Color online) Temporal evolution of the liquid-vapor interface during the evaporation process of (a) pure-water droplets and (b) salt-water droplets with a concentration of $20.5 \%$. Also shown are normalized density contours and velocity vectors (black arrows) at 5.0 ns for (c) pure-water droplets and (d) salt-water droplets with a concentration of $20.5 \%$. The density and velocity are normalized by $\sigma_{\mathrm{O}}^{-3}$ and $\left(\varepsilon_{\mathrm{O}} / m_{\mathrm{O}}\right)^{1 / 2}$, respectively.

but the noisy velocity field indicates that the salt-water droplet is not being pinned in this case.

After all the water molecules have evaporated, the $\mathrm{Na}^{+}$and $\mathrm{Cl}^{-}$ions are deposited on the surface and form salt crystals. Figure 9(a) is a snapshot of the case of salt-water droplets with a concentration of $20.5 \%$ after $6.8 \mathrm{~ns}$ of problem time: The salt is deposited as a clump in the center region of the original droplets. A side view of this deposited salt is in Fig. 9(b) and the region defined by a red circle shows a regular lattice structure that is the same as that of an ideal $\mathrm{NaCl}$ crystal [see Fig. 9(c)]. The lattice structure of $\mathrm{NaCl}$ can be regarded either as a fcc structure of one kind of ion in which the octahedral holes are occupied by the ions with opposite charge or as two interpenetrating fcc lattices made up of the two kinds of ions.

To investigate the effects of salt concentration and temperature ramp rate on the forms of salt crystals deposited, we compare the results for salt concentrations of $10.7 \%$ and $20.5 \%$ and for ramp rates of 300 and $75 \mathrm{~K} / \mathrm{ns}$ in Fig. 10. A higher concentration means more salt ions in the starting droplets and the deposited salt crystals will be larger. For the solid-liquid interaction strength parameter $\lambda=0.25$, the ramp rate does not significantly change the forms of the deposited salt crystals. All are deposited in the center region of the original droplets. The reason for this is that there is no pinned stage and no outward flow during the evaporation process, so the $\mathrm{Na}^{+}$and $\mathrm{Cl}^{-}$ions form salt crystals as the droplets slip along the surface and finally deposit in the center region as a clump.

\section{Results for solid-liquid interaction strength parameter $\lambda=0.38$}

In this section we study hydrophilic surface cases when the solid-liquid interaction strength parameter $\lambda=0.38$. Figure 11 compares the forms of the final deposited salt crystals for different salt concentrations and temperature ramp rates. We see that the salt crystals are deposited in the center of the original droplets when the ramp rate is $1500 \mathrm{~K} / \mathrm{ns}$, while the salt crystals are deposited in the region between the rim and the center of the original droplets when the ramp rate is $75 \mathrm{~K} / \mathrm{ns}$. For the higher ramp rate, a whole crystal is formed for higher salt concentrations $(20.5 \%$ and $25.1 \%)$, while several smaller crystal clumps are formed for the lower salt concentration (15.7\%). For the lower ramp rate, a complete ringlike crystal is formed for the highest salt concentration $(25.1 \%)$, while partial ringlike crystals are formed for the two other salt concentrations.

The reason for the different forms of deposited salt crystals is the different evaporation modes. Figure 12 shows the temporal evolution of the liquid-vapor interface during the evaporation process, along with the measured velocity vectors, for salt-water droplets with a concentration of $25.1 \%$. For the higher ramp rate, there are typically only two stages in the evaporation process [Fig. 12(a)]; these are the same as those described in the preceding section for salt-water droplets. When the droplets slip along the surface, there is no outward flow within the droplets [Fig. 12(c)] and the salt ions finally deposit in the center of the droplets. Depending on the salt concentration, a single clump or several small crystal clumps are formed. 
(a)

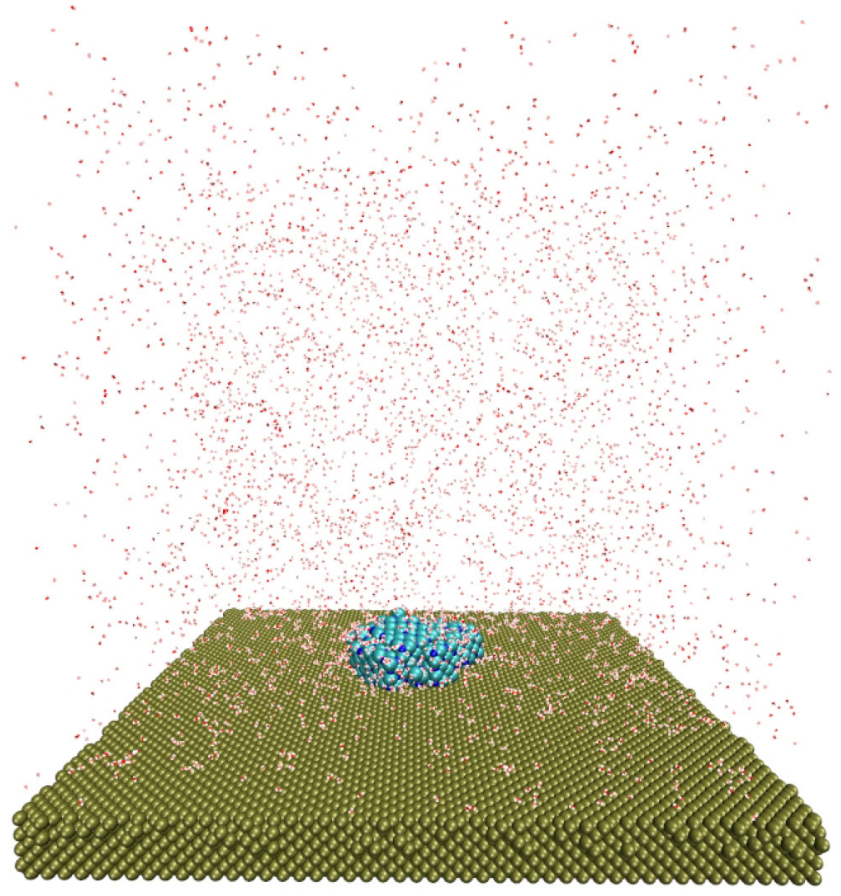

(b)

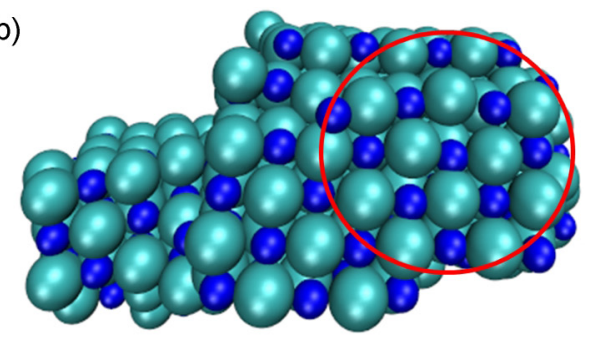

(c)

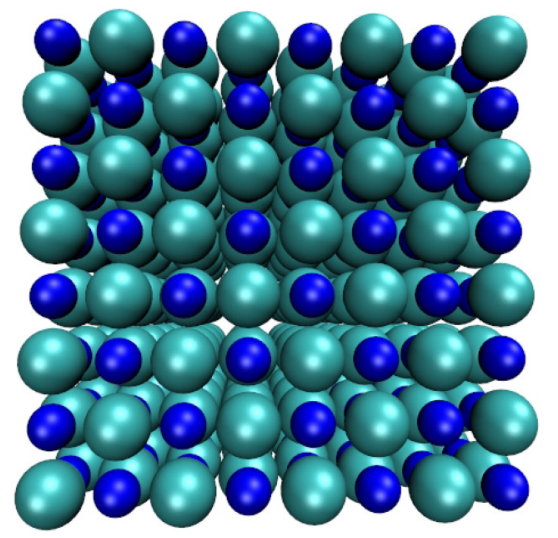

FIG. 9. (Color online) (a) Snapshot of the salt-water droplet with a concentration of $20.7 \%$ on the surface at $6.8 \mathrm{~ns}$. The red dots represent the vapor water molecules and the salt crystal is deposited on the platinumlike surface. (b) Side view of the salt crystal deposited on the surface: $\mathrm{Cl}^{-}$is cyan and $\mathrm{Na}^{+}$is blue. The red circle indicates a region with good lattice structure. (c) Lattice structure of an ideal NaCl crystal.

However, for the cases with the lower temperature ramp rate, there are typically three stages in the evaporation process [Fig. 12(b)]. The first is from 4.0 to $4.6 \mathrm{~ns}$, during which the droplets spread out a little due to the increase of temperature. In the second stage, from 4.6 to $5.4 \mathrm{~ns}$, the liquid-vapor interface is pinned at the contact line and there is a flow from the center to the rim of the droplets, as shown in Fig. 12(d). Due to this flow, some salt ions move to the droplet rim and start to form crystals. During the third stage (after $5.4 \mathrm{~ns}$ ), the droplet continues to evaporate and slip along the surface and the salt crystals finally deposit in the intermediate region of the original droplet. Depending on the salt concentration, a complete or a partial ringlike crystal can be formed.

\begin{tabular}{|c|c|c|}
\hline Salt concentration & Ramp rate: $300 \mathrm{~K} / \mathrm{ns}$ & Ramp rate: $75 \mathrm{~K} / \mathrm{ns}$ \\
\hline $10.7 \%$ & & \\
\hline $20.5 \%$ & & \\
& & \\
& & \\
\hline
\end{tabular}

FIG. 10. (Color online) Forms of the final salt crystals (top view) deposited on the solid surface with a solid-liquid interaction strength parameter $\lambda=0.25$.
To highlight the process of the formation of the salt crystals, snapshots (top view) for the case with a salt concentration of $25.1 \%$ and a ramp rate of $75 \mathrm{~K} / \mathrm{ns}$ are shown in Fig. 13. The water molecules at the start of evaporation $(t=4.0 \mathrm{~ns})$ are shown as small red dots. The salt ions are uniformly distributed within the droplets before evaporation starts [Fig. 13(a)]. In the pinned stage (from 4.6 to $5.4 \mathrm{~ns}$ ), there is a capillary flow with a characteristic velocity of $2 \mathrm{~m} / \mathrm{s}$, as shown in Fig. 12(d), so the salt ions can move outward by up to $1.6 \mathrm{~nm}$. In the

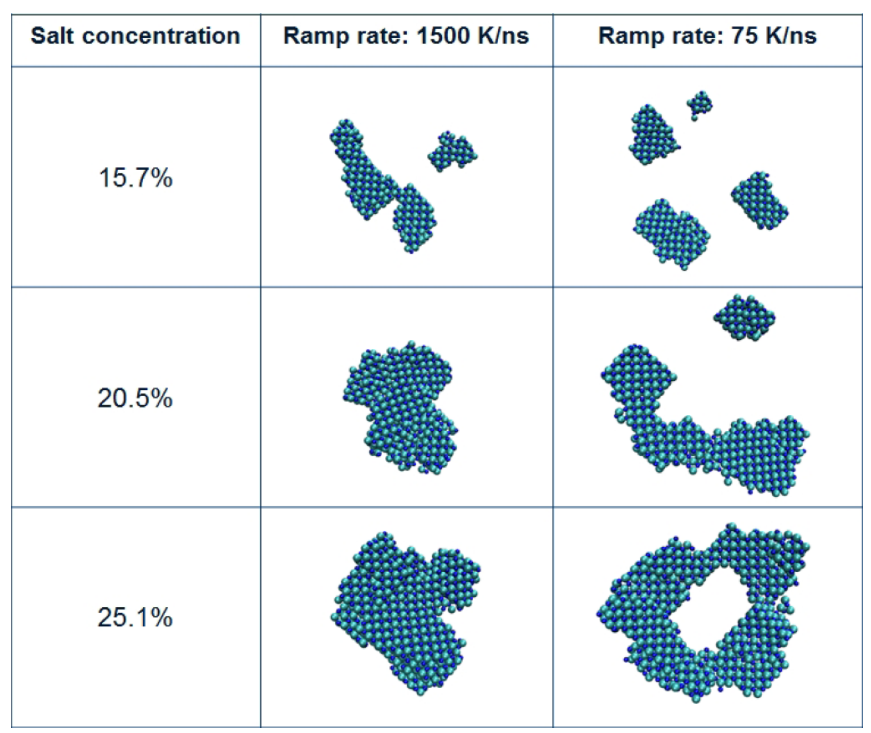

FIG. 11. (Color online) Forms of the final salt crystals (top view) deposited on the solid surface with a solid-liquid interaction strength parameter $\lambda=0.38$. 

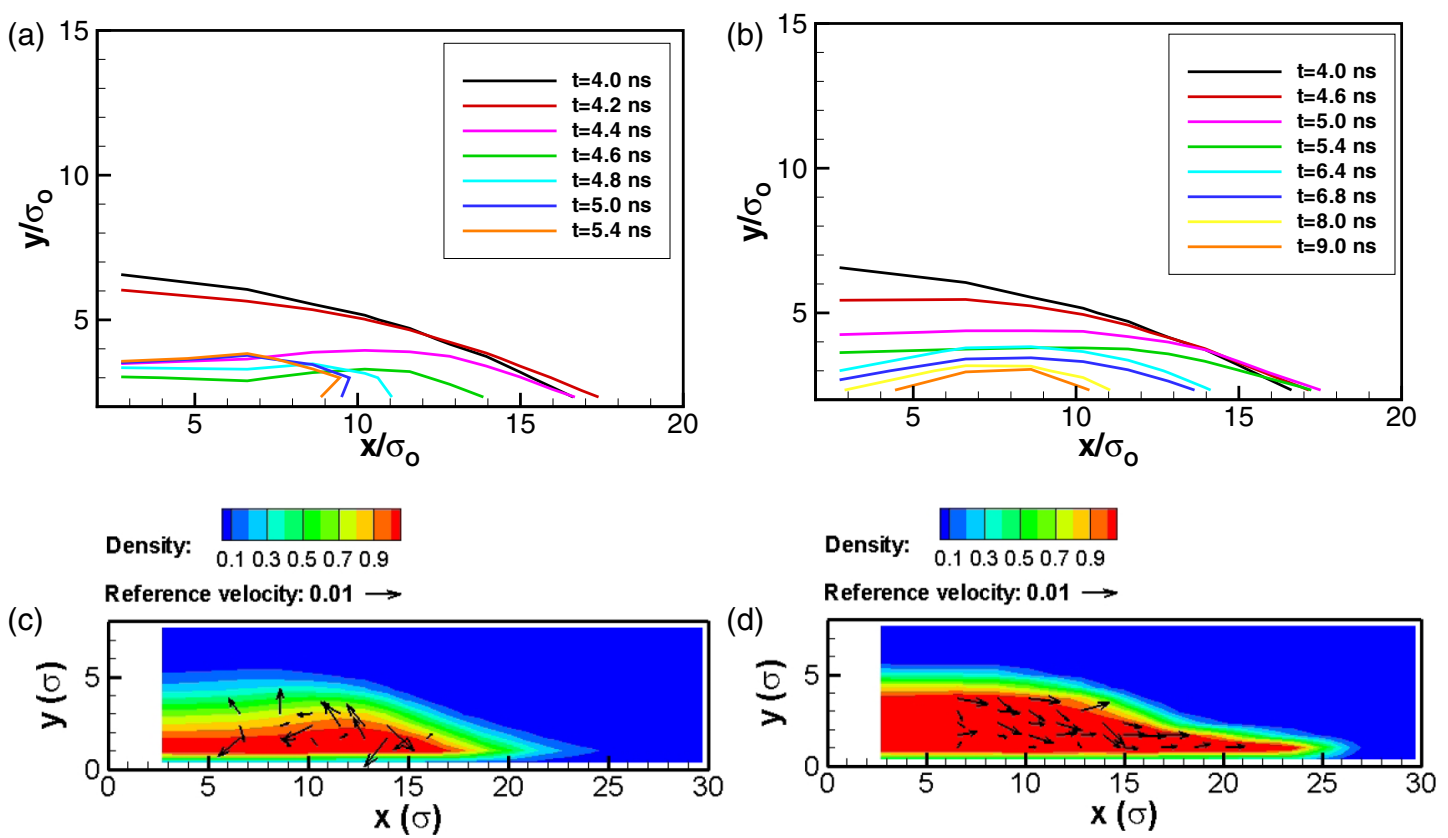

FIG. 12. (Color online) Temporal evolution of the liquid-vapor interface during the evaporation of a salt-water droplet with a concentration of $25.1 \%$ and (a) a temperature ramp rate of $1500 \mathrm{~K} / \mathrm{ns}$ and (b) a temperature ramp rate of $75 \mathrm{~K} / \mathrm{ns}$. Also shown are the normalized density contours and velocity vectors (black arrows) for (c) a ramp rate of $1500 \mathrm{~K} / \mathrm{ns}$ (snapshot at $4.4 \mathrm{~ns}$ ) and (d) a ramp rate of $75 \mathrm{~K} / \mathrm{ns}$ (snapshot at $4.8 \mathrm{~ns})$. The density and velocity are normalized by $\sigma_{\mathrm{O}}^{-3}$ and $\left(\varepsilon_{\mathrm{O}} / m_{\mathrm{O}}\right)^{1 / 2}$, respectively. The surface is hydrophilic.

region close to the rim of the droplets, some salt ions aggregate and form small clusters [Fig. 13(b)]. Once the clusters are formed, diffusion will also play an important role in the growth of clusters. The self-diffusion coefficients of $\mathrm{Na}^{+}$and $\mathrm{Cl}^{-}$ ions are of the order $10^{-9} \mathrm{~m}^{2} / \mathrm{s}$ [47]. According to Einstein's diffusion equation [48], the diffusion distance is therefore up to $1.3 \mathrm{~nm}$ in the pinned stage. So some free salt ions may reach the clusters due to diffusion and be absorbed by them. In the third stage, as the droplets shrink, the salt crystals finally deposit in the intermediate region of the original droplets, creating a ringlike form [Fig. 13(c)].

\section{CONCLUSION}

We have employed MD simulations to study the wetting and evaporation of salt-water nanodroplets on platinum surfaces. Our results show that the contact angle of saltwater nanodroplets increases with salt concentration. We have explained this phenomenon with reference to Young's equation, alongside separate MD measurements of the various surface tensions; the reason is that both the solid-liquid and liquid-vapor surface tensions increase with salt concentration. On the other hand, the evaporation rate of salt-water droplets decreases as the salt concentration increases, due to the hydration effects of salt ions.

We have observed that typically two forms of salt crystals, clump and ringlike, are deposited on the solid surface after the water molecules have fully evaporated. The forms of salt crystals are dependent on the solid-liquid interaction strength, the evaporation rate, and the initial salt concentration. For a weak solid-liquid interaction strength, all of the salt is deposited as a clump; this is because the droplet shrinks during (a)

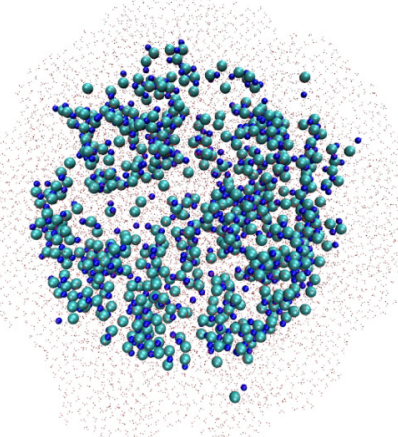

(b)

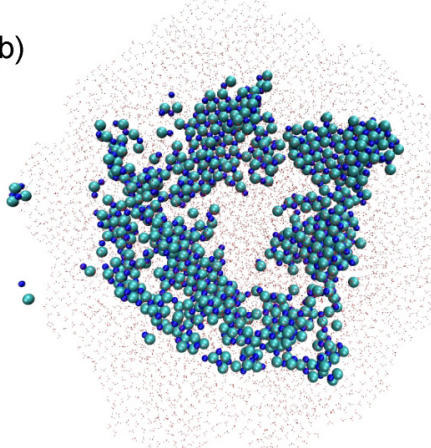

(c)

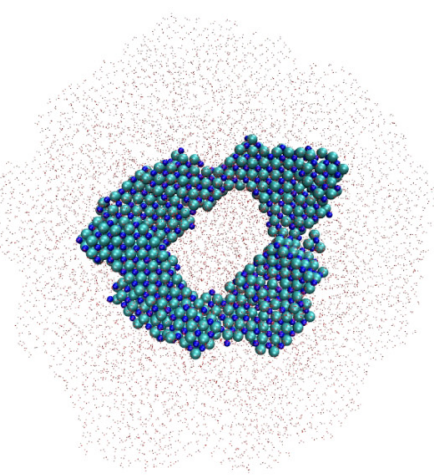

FIG. 13. (Color online) Top view of the salt ions in the droplets (salt concentration: $25.1 \%$ ) during the evaporation process (temperature ramp rate: $75 \mathrm{~K} / \mathrm{ns}$ ). The small red dots represent the water molecules before the start of evaporation. Snapshots are at (a) $t=4.0 \mathrm{~ns}$, (b) $t=$ $5.4 \mathrm{~ns}$, and (c) $t=9.0 \mathrm{~ns}$. 
evaporation. For a stronger solid-liquid interaction strength, the form of the final salt crystals is decided by the evaporation rate and the initial salt concentration. It is crucial for the formation of a ringlike crystal that there is a pinned stage during the evaporation process so that salt ions can move to the rim of the droplets. If the solid-liquid interaction strength is stronger, the evaporation rate slower, and the initial salt concentration higher, a complete ringlike salt crystal can be deposited on the surface.

In all our MD simulations, the surfaces were completely smooth at the molecular level. Investigating the evaporation of salt-water droplets on rough surfaces would be useful future work. Also of interest is whether the final deposit forms can be tuned during the evaporation process. Research on this has been reported for colloidal particles in evaporating droplets [49] and similar work on salt-water droplets is expected in the future.

\section{ACKNOWLEDGMENTS}

The research leading to these results has received funding from the United Kingdom's Engineering and Physical Sciences Research Council (EPSRC) under Grants No. EP/I011927/1 and No. EP/K038621/1. Results were obtained using the ARCHIE-WeSt High Performance Computer, under EPSRC Grants No. EP/K000586/1 and No. EP/K000195/1.
[1] H. T. El-Dessouky and H. M. Ettouney, Fundamentals of Salt Water Desalination (Elsevier, Amsterdam, 2002).

[2] M. A. Shannon, P. W. Bohn, M. Elimelech, J. G. Georgiadis, B. J. Marinas, and A. M. Mayes, Nature (London) 452, 301 (2008).

[3] A. S. Goudie and H. A. Viles, Salt Weathering Hazards (Wiley, Chichester, 1997).

[4] N. Sghaier, M. Prat, and S. Ben Nasrallah, Chem. Eng. J. 122, 47 (2006).

[5] N. Shahidzadeh-Bonn, S. Rafai, D. Bonn, and G. Wegdam, Langmuir 24, 8599 (2008).

[6] N. Shahidzadeh, M. F. L. Schut, J. Desarnaud, M. Prat, and D. Bonn, Sci. Rep. 5, 10335 (2015).

[7] T. Werder, J. H. Walther, R. L. Jaffe, T. Halicioglu, and P. Koumoutsakos, J. Phys. Chem. B 107, 1345 (2003).

[8] K. Ritos, N. Dongari, M. K. Borg, Y. H. Zhang, and J. M. Reese, Langmuir 29, 6936 (2013).

[9] J. G. Zhang, F. Leroy, and F. Muller-Plathe, Langmuir 29, 9770 (2013).

[10] J. G. Zhang, F. Leroy, and F. Muller-Plathe, Phys. Rev. Lett. 113, 046101 (2014).

[11] W. K. Chen, J. Koplik, and I. Kretzschmar, Phys. Rev. E 87, $052404(2013)$

[12] C. D. Daub, D. Bratko, and A. Luzar, J. Phys. Chem. C 115, 22393 (2011).

[13] C. Caleman and D. van der Spoel, Phys. Chem. Chem. Phys. 9 , 5105 (2007).

[14] B. B. Wang, X. D. Wang, M. Chen, and J. L. Xu, Entropy 15, 1232 (2013).

[15] G. B. Macpherson and J. M. Reese, Mol. Simul. 34, 97 (2008).

[16] M. K. Borg, G. B. Macpherson, and J. M. Reese, Mol. Simul. 36, 745 (2010).

[17] W. D. Nicholls, M. K. Borg, D. A. Lockerby, and J. M. Reese, Mol. Simul. 38, 781 (2012).

[18] M. K. Borg, D. A. Lockerby, and J. M. Reese, J. Comput. Phys. 233, 400 (2013).

[19] M. K. Borg, D. A. Lockerby, and J. M. Reese, J. Comput. Phys. 255, 149 (2013).

[20] www.openfoam.org

[21] M. K. Borg, D. A. Lockerby, and J. M. Reese, J. Chem. Phys. 140, 074110 (2014).

[22] J. L. F. Abascal and C. Vega, J. Chem. Phys. 123, 234505 (2005).

[23] J. Alejandre, G. A. Chapela, F. Bresme, and J. P. Hansen, J. Chem. Phys. 130, 174505 (2009).
[24] P. M. Agrawal, B. M. Rice, and D. L. Thompson, Surf. Sci. 515, 21 (2002).

[25] M. J. P. Nijmeijer, C. Bruin, A. F. Bakker, and J. M. J. van Leeuwen, Phys. Rev. A 42, 6052 (1990).

[26] D. Surblys, Y. Yamaguchi, K. Kuroda, M. Kagawa, T. Nakajima, and H. Fujimura, J. Chem. Phys. 140, 034505 (2014).

[27] J. Alejandre and G. A. Chapela, J. Chem. Phys. 132, 014701 (2010).

[28] E. W. Washburn, International Critical Tables of Numerical Data, Physics, Chemistry and Technology (McGraw-Hill, New York, 1928), Vol. IV.

[29] L. G. MacDowell, J. Benet, and N. A. Katcho, Phys. Rev. Lett. 111, 047802 (2013).

[30] R. de Gregorio, J. Benet, N. A. Katcho, F. J. Blas, and L. G. MacDowell, J. Chem. Phys. 136, 104703 (2012).

[31] N. Tretyakov, M. Muller, D. Todorova, and U. Thiele, J. Chem. Phys. 138, 064905 (2013).

[32] J. W. Gibbs, The Collected Works of J. Willard Gibbs (Yale University Press, London, 1957).

[33] J. H. Weijs, A. Marchand, B. Andreotti, D. Lohse, and J. H. Snoeijer, Phys. Fluids 23, 022001 (2011).

[34] T. Ingebrigtsen and S. Toxvaerd, J. Phys. Chem. C 111, 8518 (2007).

[35] R. J. Good and M. N. Koo, J. Colloid Interface Sci. 71, 283 (1979).

[36] L. Boruvka and A. W. Neumann, J. Chem. Phys. 66, 5464 (1977).

[37] M. H. Factorovich, V. Molinero, and D. A. Scherlis, J. Am. Chem. Soc. 136, 4508 (2014)

[38] C. A. Ward and J. Wu, Phys. Rev. Lett. 100, 256103 (2008).

[39] C. A. Ward and K. Sefiane, Adv. Colloid Interface Sci. 161, 171 (2010).

[40] R. D. Deegan, O. Bakajin, T. F. Dupont, G. Huber, S. R. Nagel, and T. A. Witten, Nature (London) 389, 827 (1997).

[41] R. D. Deegan, Phys. Rev. E 61, 475 (2000).

[42] R. D. Deegan, O. Bakajin, T. F. Dupont, G. Huber, S. R. Nagel, and T. A. Witten, Phys. Rev. E 62, 756 (2000).

[43] S. Maruyama, S. Matsumoto, and A. Ogita, Therm. Sci. Eng. 2, 77 (1994).

[44] H. Kohler, Trans. Faraday Soc. 32, 1152 (1936).

[45] K. S. Birdi, D. T. Vu, and A. Winter, J. Phys. Chem. 93, 3702 (1989). 
[46] S. M. Rowan, M. I. Newton, and G. McHale, J. Phys. Chem. 99, 13268 (1995).

[47] A. Ghaffari and A. Rahbar-Kelishami, J. Mol. Liq. 187, 238 (2013).
[48] J. P. Boon and S. Yip, Molecular Hydrodynamics (McGraw-Hill, New York, 1980)

[49] R. Bhardwaj, X. H. Fang, P. Somasundaran, and D. Attinger, Langmuir 26, 7833 (2010). 